\title{
ADDENDUM
}

\section{Interception of teicoplanin oxidation intermediates yields new antimicrobial scaffolds}

\author{
Yu-Chen Liu, Yi-Shan Li, Syue-Yi Lyu, Li-Jen Hsu, Yu-Hou Chen, Yu-Ting Huang, Hsiu-Chien Chan, Chuen-Jiuan Huang, \\ Gan-Hong Chen, Chia-Cheng Chou, Ming-Daw Tsai \& Tsung-Lin Li
}

Nat. Chem. Biol. 7, 304-309 (2011); published online 10 April 2011; addendum published after print 17 April 2015

We have determined that the original crystal structure in our paper (Protein Data Bank (PDB) code 2WDX) was not of sufficient quality to provide strong support for a diol intermediate, although the mechanistic conclusions of the paper, which were also based on mutational and biochemical analysis, still stand. At the urging of our colleagues, we have replaced this structure with a second structure (PDB code $4 \mathrm{~K} 3 \mathrm{~T}$ ), solved as follows: Dbv29 protein in the buffer of $20 \mathrm{mM}$ Tris- $\mathrm{HCl}, \mathrm{pH} 8.0$, and $100 \mathrm{mM} \mathrm{NaCl}$ was concentrated to $10 \mathrm{mg} \mathrm{ml}^{-1}$. Dbv29 ligand-free crystals were grown under crystallization conditions of $0.2 \mathrm{M}$ diammonium hydrogen citrate and 17\% (w/v) PEG3350, optimized through thousands of screening conditions using Mosquito HTS liquid handler (TTP Labtech Ltd, Hertfordshire, UK). Bigger integral crystals were singled out and soaked with different concentrations of teicoplanin versus various soaking time courses (10 s-24 h). Diffraction data of teicoplanin-soaked crystals were collected at beamlines 13B1, 13C1 and 15A1 of NSRRC (Taiwan) and beamlines 12B2 and 44XU of SPring-8 (Japan). Corresponding data sets were indexed and scaled by HKL2000 package (http://www.hkl-xray.com/hkl-package/). Soaking the protein crystals with $40.5 \mathrm{mM}$ teicoplanin for $1.2 \mathrm{~h}$ was determined to be the best condition for the formation of ternary complexes. Structures were solved by the molecular replacement method using the coordinates of native Dbv29 (PDB code 2WDW) as the search model and were refined by REFMAC (http://www.ccp4.ac.uk/) and PHENIX (http://www.phenix-online.org/). Ligands were identified by the difference $\left(F_{\mathrm{o}}-F_{\mathrm{c}}\right)$ electron density maps and built using the program Coot (http://www2.mrc-lmb.cam.ac.uk/personal/pemsley/coot/). Occupancy refinement was performed using PHENIX to determine the occupancy of ligands. The best-complexed structure was obtained at 1.93-Å resolution (Fig. 1). The structure quality was further checked by the Uppsala Electron Density Server (EDS; http://eds.bmc.uu.se/) and Twilight (a tool for ligand density validation; http://www.ruppweb.org/twilight/). The detailed data collection and refinement statistics are shown in Table 1.

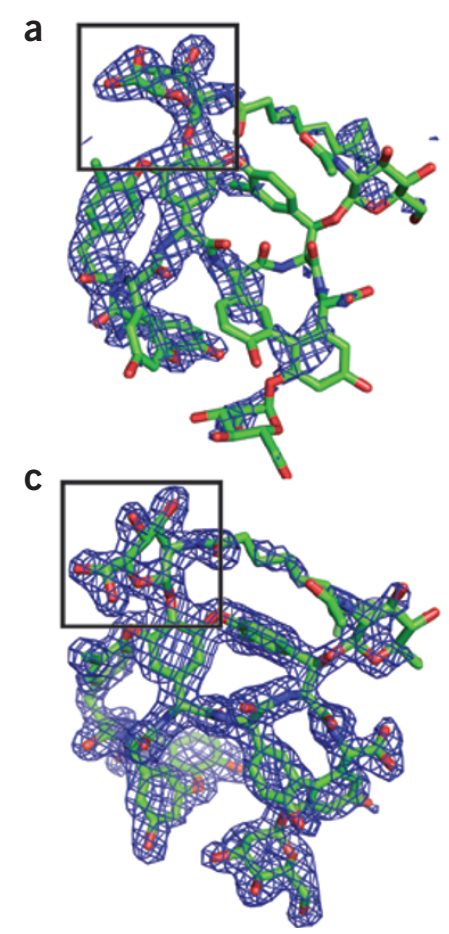

b

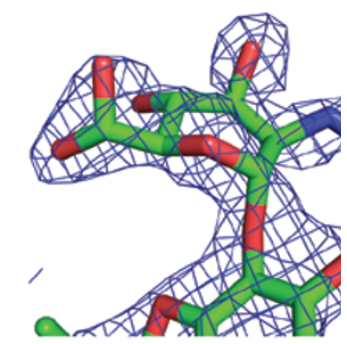

d

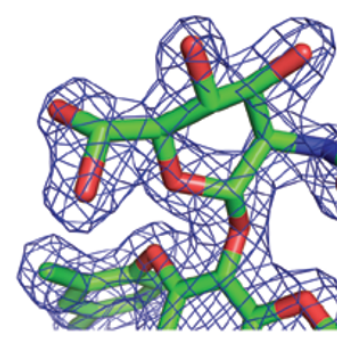

Figure 1 | Models and electron density maps of the teicoplanin diol intermediate. (a) $2 F_{0}-F_{c}$ electron density map of the teicoplanin diol intermediate bound at the active site contoured at $1 \sigma$ (PDB code 4K3T, chain I). (b) Close-up view of the $N$-acyl glucosaminyl moiety of the teicoplanin diol intermediate in a. (c) $2 F_{o}-F_{c}$ electron density map of the teicoplanin diol intermediate located between enzymes contoured at $1 \sigma$ (PDB code 4K3T; chain M). (d) Close-up view of the $N$-acyl glucosaminyl moiety of the teicoplanin diol intermediate in $\mathbf{c}$.
Table 1 | Data collection and refinement statistics for Dbv29-teicoplanin structure.

\begin{tabular}{ll} 
& Dbv29-teicoplanin \\
\hline $\begin{array}{l}\text { Data collection } \\
\text { Space group }\end{array}$ & $P 2_{1}$ \\
Cell dimensions & \\
$a, b, c(\AA)$ & $61.79,150.78,124.85$ \\
$\alpha, \beta, \gamma\left(^{\circ}\right)$ & $90.00,98.40,90.00$ \\
Resolution $(\AA)$ & $30.00-1.93(1.96-1.93)$ \\
$R_{\text {merge }}$ & $9.1(57.3)$ \\
$I / \sigma I$ & $15.0(2.9)$ \\
Completeness $(\%)$ & $97.8(97.1)$ \\
Redundancy & $4.6(4.6)$ \\
Wavelength $(\AA)$ & 1 \\
Temperature $(K)$ & 100 \\
Refinement &
\end{tabular}

Refinement

Resolution $(\AA) \quad 1.93$

No. reflections $\quad 155,629$

$R_{\text {work }} / R_{\text {free }} \quad 0.160 / 0.205$

No. atoms

Protein 16,104

Ligand/ion 615

Water $\quad 1,400$

$\begin{array}{ll}B \text {-factors } & \\ \text { Protein } & 30.9\end{array}$

Ligand/ion $\quad 57.7$

Water $\quad 37.7$

r.m.s. deviation values

Bond lengths $(\AA)$

Bond angles $\left(^{\circ}\right) \quad 2.030$

PDB code 4K3T

Values in parentheses are for the highest-resolution shell. 